\title{
Two severe cases of H7N9 pneumonia patients with immunoneuroendocrine axis dysfunction and vitamin D insufficiency
}

\author{
Jin Yao', Linhua Liu', Gang Chen ${ }^{2}$ and Leng Lin ${ }^{1,3^{*}}$
}

\begin{abstract}
Background: The immunoneuroendocrine axis plays a major role in the regulation of the host's response to infection, but its role in severe H7N9 pneumonia is still unknown. Therefore, this study is carried out to explore the relationship between the immunoneuroendocrine axis and severe H7N9 pneumonia.

Case presentantion: The study included two H7N9 pneumonia patients. Endocrine response and cellular immune function in prolonged phase of these two severe H7N9 pneumonia cases were reported and analyzed. A 57-year-old male patient (case 1) and a 68-year-old male patient (case 2) were admitted because of cough, fever and dyspnea. Moist rales were present in both lungs. The rest of the examination was reportedly normal. The laboratory test showed that (1) The patients had loss of cortisol rhythm and elevated cortisol level at $4 \mathrm{pm}$. (2) The patients showed decline of cellular immune function. (3) The patients showed vitamin D insufficiency. (4) Case 2 had higher cortisol level but lower DHEAS, serum phosphorus and vitamin D level as well as cellular immune function than case 1. (5) The thyroid axis, gonadal and lactotropic axis were normal, so were the level of FT3, FT4, STSH and LH, FSH, T, E2 as well as PRL in these two cases. Chest CT revealed inflammation of both lungs especially in right lung. Real time RT-PCR by Centers for Disease Control and Prevention (CDC) confirmed H7N9 infection.
\end{abstract}

Conclusion: Immunoneuroendocrine axis dysfunction may play an important role in severe H7N9 pneumonia. We need pay more attention to hypophosphatemia and vitamin D insufficiency in H7N9 pneumonia.

Keywords: H7N9 pneumonia, Vitamin D insufficiency, Immunoneuroendocrine axis

\section{Background}

Since the first human infection with influenza A (H7N9) viruses has been identified in Shanghai on March 31, 2013, the latest variant of the avian flu virus has spread across ten provinces in China. Clinical data have been collected to study the immunoneuroendocrine axis in prolong phase of severe H7N9 pneumonia since two H7N9 pneumonia patients were admitted to Fujian Provincial Hospital on April 29,2013. To our knowledge, no study had specially addressed the role of immunoneuroendocrine axis in prolong phase of severe H7N9 pneumonia.

\footnotetext{
*Correspondence: Lenglin_fz@163.com

'Department of Infectious Disease, Fujian Provincial Hospital, Fujian Medical University, Fuzhou 350001, China

${ }^{3}$ Department of Infectious Disease, Fujian Provincial Hospital, Fujian Medical University, Box 4-704, 92 Huqian Road, Fuzhou, Fujian, China

Full list of author information is available at the end of the article
}

\section{Case presentation}

A 57-year-old male patient (case 1) and a 68-year-old male patient (case 2) were admitted because of cough, fever and dyspnea. It is reported that they had a history of contact with poultry. Case 1 had been well before admission and so was Case 2 except for some symptoms of coronary disease and chronic obstructive pulmonary disease (COPD) before admission. On examination, the temperature of case 1 and case 2 were $39^{\circ} \mathrm{C}$ and $39.7^{\circ} \mathrm{C}$ respectively, pulse were 85 beats/min and 78 beats/min respectively, respiratory rate were 25 breaths/min and 30 breaths/min respectively, and blood pressure were 143/76 $\mathrm{mmHg}$ and 112/78 $\mathrm{mmHg}$ respectively. The patients were alert, oriented but appeared fatigued and sick. They were in shortness of breath and with cyanotic lips. Moreover, moist rales were present in both lungs. Chest CT revealed inflammation of both lungs especially in right lung. While, the rest of the examination was reportedly normal. The laboratory test was shown in 
Table 1 Laboratory data of study subjects

\begin{tabular}{|c|c|c|c|}
\hline Laoratory data & Case 1 & Case2 & Normal range \\
\hline \multicolumn{4}{|l|}{ Blood cell count } \\
\hline WBC $(\times 109 / L)$ & $2.0 \times 10^{9}$ & $2.7 \times 10^{9}$ & $4-10$ \\
\hline N (\%) & 68 & 67 & $50-75$ \\
\hline L (\%) & 22 & 23 & $20-40$ \\
\hline \multicolumn{4}{|l|}{ Blood levels of electrolytes } \\
\hline Serum sodium (mmol/L) & 131 & 125 & $135-145$ \\
\hline Serum potassium (mmol/L) & 4.8 & 4.4 & $3.5-5.5$ \\
\hline Corrected serum calcium (mmol/L) & 2.13 & 2.27 & $2.25-2.65$ \\
\hline Serum phosphorus (mmol/L) & 1.04 & 0.55 & $0.80-1.48$ \\
\hline Serum magnesium (mmol/L) & 0.95 & 0.85 & $0.6-1.2$ \\
\hline \multicolumn{4}{|l|}{ Renal-function tests } \\
\hline Serum uric acid (mmol/L) & 163 & 106 & 237-357 (male) \\
\hline Creatinine (umol/L) & 67 & 73 & $40-135$ \\
\hline BUN (mmol/L) & 4.1 & 5.3 & $2.1-7.1$ \\
\hline \multicolumn{4}{|l|}{ Liver-function tests } \\
\hline $\mathrm{ALT}(\mathrm{U} / \mathrm{L})$ & 83 & 16 & $5-40$ \\
\hline AST (U/L) & 83 & 47 & $5-42$ \\
\hline Albumin $(\mathrm{g} / \mathrm{l})$ & 35 & 27 & $35-55$ \\
\hline$D$ dimmer $(\mu \mathrm{g} / \mathrm{ml})$ & 2.4 & 2.6 & $<0.5$ \\
\hline \multicolumn{4}{|l|}{ Blood gas analysis } \\
\hline $\mathrm{PH}$ & 7.38 & 7.47 & $7.35-7.45$ \\
\hline $\mathrm{PCO}_{2}(\mathrm{mmHg})$ & 37.1 & 27 & $35-45$ \\
\hline $\mathrm{PO}_{2}(\mathrm{mmHg})$ & 57.7 & 63 & $90-100$ \\
\hline \multicolumn{4}{|l|}{ Cellular immune function } \\
\hline CD3 (\%) & 48 & 48 & $55-84$ \\
\hline CD3 (/UI) & 440 & 273 & $690-2540$ \\
\hline CD4 (\%) & 30 & 21 & $31-60$ \\
\hline CD4 (/UI) & 279 & 120 & $410-1590$ \\
\hline CD8 (\%) & 16 & 26 & $13-41$ \\
\hline CD8 (/UI) & 152 & 148 & $190-1140$ \\
\hline CD4/CD8 & 1.88 & 0.81 & $1.05-2.03$ \\
\hline NK (\%) & 25 & 30 & $5-27$ \\
\hline NK (/UI) & 256 & 201 & $90-590$ \\
\hline Fasting blood glucose (mmol/l) & 6.8 & 4.8 & $4.0-5.5$ \\
\hline \multicolumn{4}{|l|}{ Thyroid axis } \\
\hline FT3 (pmol/l) & 4.94 & 3.37 & $3.1-6.8$ \\
\hline FT4 (pmol/l) & 21.15 & 21.98 & $12-22$ \\
\hline sTSH (mIU/l) & 0.85 & 0.37 & $0.27-4.2$ \\
\hline \multicolumn{4}{|l|}{ Lactotropic axis } \\
\hline Prolactin (ng/ml) & 8.79 & 11.13 & $1.64-13.13$ \\
\hline
\end{tabular}

Table 1 Laboratory data of study subjects (Continued)

\begin{tabular}{lccc}
\hline Gonadal axis & & & \\
$\mathrm{LH}(\mathrm{IU} / \mathrm{l})$ & 8.27 & 10.96 & $1.25-8.62$ \\
$\mathrm{FSH}(\mathrm{IU} / \mathrm{l})$ & 8.0 & 21.1 & $1.27-19.26$ \\
$\mathrm{~T}(\mathrm{nmmol} / \mathrm{l})$ & 21.2 & 8.69 & $6.07-27.24$ \\
$\mathrm{E} 2(\mathrm{pg} / \mathrm{ml})$ & 46 & 21 & $20-47$ \\
Adrenal axis & & & \\
ACTH $8 \mathrm{am}(\mathrm{pg} / \mathrm{ml})$ & 27.5 & 22.2 & $7.2-63.6$ \\
Cortisol $8 \mathrm{am}(\mathrm{nmol} / \mathrm{l})$ & 364 & 488 & $240-680$ \\
Cortisol 4 pm (nmol/l) & 307.8 & 406.5 & $<276$ \\
DHEAS (ug/dl) & 167.6 & 45.4 & $38-313$ \\
Parathyroid hormone $(\mathrm{Pg} / \mathrm{ml})$ & 5.3 & 36.7 & $15-88$ \\
25OH-VitD $(\mathrm{ng} / \mathrm{ml})$ & 29 & 13.9 & $30-100$ \\
Procalcitonin $(\mathrm{ng} / \mathrm{ml})$ & 0.31 & 0.26 & $<0.05$ \\
$\begin{array}{l}\text { Erythrocyte sedimentation } \\
\text { rate }(\mathrm{mm} / \mathrm{h})\end{array}$ & 17 & 22 & $0-15$ \\
\hline
\end{tabular}

Table 1. RNA was extracted from throat-swab samples with the RNeasy mini kit (Qiagen, Valencia, CA, USA) as per the manufacturer's protocol and tested by real-time RT-PCR withH7N9-specific primers and probes as previously described [1]. The specific sequences have been published on the WHO website at http://www.who.int/influenza/gisrs_laboratory/a_h7n9/en/. Real time RT-PCR by CDC confirmed H7N9 infection. The functions of thyroid, adrenal, gonad and cellular immune system, and the levels of PRL, PTH, 25OH-VitD were examined one week after methylprednisolone administration stopped. The immunoneuroendocrine axis test showed that (1) The patients had loss of cortisol rhythm and elevated cortisol level at $4 \mathrm{pm}$. (2) The patients showed decline of cellular immune function. (3) The patients showed vitamin D insufficiency. (4) Case 2 had higher cortisol level but lower DHEAS, serum phosphorus and vitamin D level as well as cellular immune function than case 1. (5) The thyroid axis, gonadal and lactotropic axis were normal, so were the level of FT3, FT4, STSH and LH, FSH, T, E2 as well as PRL in these two cases. Two patients were diagnosed severe H7N9 pneumonia with mild acute respiratory distress syndrome. The patients received antiviral treatment with oseltamivir and cefoperazone was used to protect against infection. They were also administered methylprednisolone $40 \mathrm{mg} / \mathrm{d}$ for 3 days. Noninvasive mechanical ventilation, thymalfasin, rocalirol and supportive treatment were provided as well. The patients were discharged from hospital after three weeks.

\section{Conclusion}

In our study, the two cases showed the loss of cortisol rhythm, elevated cortisol level at $4 \mathrm{pm}$ and decline of cellular immune function in prolonged phase of H7N9 pneumonia. Interestingly, case 2 had higher cortisol level 
and lower DHEAS levels than case 1, but had lower cellular immune function. One meta-analysis also has demonstrated the association between high cortisol levels and mortality, which made cortisol an useful biomarker for assessing prognosis in patients with severe communityacquired pneumonia (CAP) [2]. Glucocorticoids influence the traffic of circulating leukocytes and inhibit many functions of leukocytes and immune accessory cells [3]. They inhibit cell accumulation at inflammatory sites and reduce the number of circulating lymphocytes, monocytes, and eosinophils by inducing cell apoptosis [4]. Conversely, cytokines, produced by activated immune cells and neuroendocrine cells as well, are able to modulate the hypothalamus-pituitary-adrenal (HPA) axis at each level: the hypothalamus, pituitary, and adrenal glands [5]. DHEAS is a pleiotropic adrenal hormone, primarily regulated by corticotropin, with proimmune and proinflammatory effects, opposing the immunosuppressive effects of glucocorticoids. The high glucocorticoid level and low DHEAS level suggest an imbalance between immunosuppressive and immunostimulatory adrenocortical hormones, which can result in increased susceptibility to infectious complications during the chronic phase of severe illness.

The level of vitamin D in the two patients were all under normal range and case 2 showed hypophosphatemia. Although some studies had shown that vitamin D insufficiency and hypophosphatemia might weaken the host's immune defense [6,7], whether or not low level of serum phosphorus and vitamin D would lead to adverse outcome in H7N9 remained unknown. Therefore further studies should be conducted to answer the aforementioned question. One limitation of this study was that we didn't know whether our patients had low level of $25 \mathrm{OH}-\mathrm{VitD}$ and serum phosphorus before they had H7N9 pneumonia. But we did need pay more attention to hypophosphatemia and vitamin D insufficiency in severe H7N9 pneumonia. Thus, we used rocalirol to correct vitamin D deficiency in our patient. In our opinion, vitamin D should be measured in severe H7N9 Pneumonia.

The thyroid axis, gonadal and lactotropic axis were normal, so were the level of FT3, FT4, STSH and LH, FSH, T, E2 as well as PRL in these two cases we studied because they were in prolonged but not acute phase of infection.

In conclusion, our cases report suggested that immunoneuroendocrine axis dysfunction might play an important role in severe H7N9 pneumonia. We need pay more attention to hypophosphatemia and vitamin D insufficiency in H7N9 pneumonia.

\section{Consent}

Written informed consent was obtained from the patient for publication of this case report and any accompanying images. A copy of the written consent is available for review by the Editor of this journal. This study was proved by IRB of Fujian Provincial Hospital.

\section{Competing interests}

The authors declare that they have no competing interests.

\section{Authors' contributions}

$J Y$ participated in analysis, interpretation of data and wrote the manuscript. Li $L$ and GC participated in acquisition of data and design. Le $L$ participated in design, interpretation of data and gave final approval of the version to be published. All authors read and approved the final manuscript.

\section{Acknowledgements}

This study was supported by China's National Natural Science Fund (81270874).

\section{Author details}

${ }^{1}$ Department of Infectious Disease, Fujian Provincial Hospital, Fujian Medical University, Fuzhou 350001, China. ${ }^{2}$ Department of Endocrinology, Fujian Provincial Hospital Key Laboratory of Endocrinology, Fujian Medical University, Fuzhou 350001, China. ${ }^{3}$ Department of Infectious Disease, Fujian Provincial Hospital, Fujian Medical University, Box 4-704, 92 Huqian Road, Fuzhou, Fujian, China.

Received: 2 October 2013 Accepted: 18 January 2014

Published: 28 January 2014

\section{References}

1. Gao RB, Cao B, Hu YW, Feng ZJ, Wang DY, Hu WF, Chen J, Jie ZJ, Qiu H, Xu K, Xu XW, Lu HZ, Zhu WF, Gao ZC, Xiang NJ, Shen YH, He ZB, Gu Y, Zhang ZY, Yang Y, Zhao X, Zhou L, Li XD, Zou SM, Zhang Y, Li XY, Yang L, Guo JF, Dong J, Li Q, et al: Human Infection with a Novel Avian-Origin Influenza A (H7N9) Virus. N Engl J Med 2013; 368: 1888-1897.

2. Salluh Jl, Shinotsuka CR, Soares M, Bozza FA, Lapa e Silva JR, Tura BR, Bozza PT, Vidal CG: Cortisol levels and adrenal response in severe communityacquired pneumonia: a systematic review of the literature. J Crit Care 2010, 25:541. e1-8.

3. Munck A, Guyre PM, Holbrook NJ: Physiological functions of glucocorticoids in stress and their relation to pharmacological actions. Endocr Rev 1984, 5:25-44

4. Amsterdam A, Tajima K, Sasson R: Cell-specific regulation of apoptosis by glucocorticoids: implication to their anti-inflammatory action. Biochem Pharmacol 2002, 64:843-850.

5. Imura H, Fukata J: Endocrine-paracrine interaction in communication between the immune and endocrine systems. Activation of the hypothalamicpituitary-adrenal axis in inflammation. Eur J Endocrinol 1994, 130:32-37.

6. Aregbesola A, Voutilainen S, Nurmi T, Virtanen JK, Ronkainen K, Tuomainen TP: Serum 25-hydroxyvitamin D3 and the risk of pneumonia in an ageing general population. J Epidemiol Community Health 2013, 67:533-536.

7. Haglin LM, Burman LA, Nilsson M: Predisposing chronic diseases and hypophosphatemia in patients with influenza. Arch Gerontol Geriatr 2010, 51:26-30.

\section{doi:10.1186/1471-2334-14-44}

Cite this article as: Yao et al:: Two severe cases of H7N9 pneumonia patients with immunoneuroendocrine axis dysfunction and vitamin D insufficiency. BMC Infectious Diseases 2014 14:44. 